\title{
INDUCED MAPPINGS OF HOMOLOGY DECOMPOSITIONS
}

\author{
MARTIN ARKOWITZ \\ Mathematics Department, Dartmouth College \\ Hanover, New Hampshire 03755, U.S.A. \\ E-mail: Martin.Arkowitz@Dartmouth.edu
}

\begin{abstract}
We give conditions for a map of spaces to induce maps of the homology decompositions of the spaces which are compatible with the homology sections and dual Postnikov invariants. Several applications of this result are obtained. We show how the homotopy type of the $(n+1)$ st homology section depends on the homotopy type of the $n$th homology section and the $(n+1)$ st homology group. We prove that all homology sections of a co-H-space are co$\mathrm{H}$-spaces, all $n$-equivalences of the homology decomposition are co-H-maps and, under certain restrictions, all dual Postnikov invariants are co-H-maps. We give a new proof of a result of Berstein and Hilton which gives conditions for a co-H-space to be a suspension.
\end{abstract}

1. Introduction. The Postnikov decomposition of a 1-connected space has been extremely useful in homotopy theory. A basic property of this construction is the existence of induced maps, that is, a map between spaces induces maps between the Postnikov sections of the spaces which are compatible with all the data of the Postnikov decompositions [Wh, Chap. IX]. This can be used, for example, to show that the Postnikov sections of an H-space are H-spaces and the Postnikov invariants are H-maps. The Eckmann-Hilton dual of the Postnikov decomposition of a space is the homology decomposition of a space. This too has been a very useful way to describe a space. However, it has been known for some time that induced maps of homology decompositions do not always exist. In $\left[\mathrm{Cu}_{1}\right]$ Curjel gives necessary and sufficient conditions for a map of spaces to induce compatible maps of homology sections. Here we carry this one step further by giving conditions for the induced maps to be compatible with the dual Postnikov invariants. We derive several consequences of these results. We show that, with certain restrictions, the homotopy type of the homology sections of a space are determined by the homotopy type of the space. We also give conditions under which we can describe the homotopy type of $(n+1)$ st homology sections with fixed $n$th homology section and fixed $(n+1)$ st homology group.

1991 Mathematics Subject Classification: Primary 55P30; Secondary 55P45, 55S99.

The paper is in final form and no version of it will be published elsewhere. 
In the last section we consider the homology decomposition of a co-H-space $X$. We prove that the homology sections are co-H-spaces which are compatible with the co-H-structure of $X$ and, if $X$ is either 2-connected or has torsion-free homology, that the dual Postnikov invariants are co-H-maps. From this we obtain a new proof of the following result of Berstein and Hilton: a $(q-1)$-connected co-H-space of dimension $\leq 3 q-3$ is equivalent to a suspension.

For the remainder of this section we present our notation and conventions. All spaces are 1-connected, based spaces of the based homotopy type of a CW-complex. We denote the base point of a space and the one point space by $*$, the constant map by 0 and the identity map or homomorphism by $i d$. All maps preserve the base point and we do not distinguish notationally between a map and its homotopy class. Thus equality of maps means either homotopy of the maps or equality of their homotopy classes. The usual notation of homotopy theory will be in effect: $[A, B]$ for the set of homotopy classes $A \rightarrow B, f_{*}:[A, B] \rightarrow\left[A, B^{\prime}\right]$ for the function induced by $f: B \rightarrow B^{\prime}, \Sigma$ for the reduced suspension, $C_{g}$ for the mapping cone of a map $g, K(G, n)$ for the Eilenberg-MacLane space of type $(G, n)$ and $M(G, n)$ for the Moore space of type $(G, n)$. We note that $M(G, n)$ can be regarded as a $\mathrm{CW}$-complex of dimension $\leq n+1$ which has dimension $\leq n$ when $G$ is free-abelian. The $n$th homotopy group $\pi_{n}(G ; X)$ of $X$ with coefficients in $G$ is $[M(G, n), X]$. The $n$th cohomology group $H^{n}(X ; G)$ of $X$ with coefficients in $G$ will often be taken to be $[X, K(G, n)]$.

We would like to thank Peter Hilton and Jin-Yen Tai for valuable discussions. We are grateful to Marek Golasiński for providing us with a copy of [G-K].

2. Basic classes and homology decompositions. We begin with a statement of the generalized Blakers-Massey Theorem which follows easily from $\left[\mathrm{Hi}_{2}\right.$, Thm. $\left.1^{\prime}\right]$.

TheOrem 2.1. Let $A \stackrel{i}{\longrightarrow} Y \stackrel{p}{\longrightarrow} C$ be a cofibre sequence with $A(m-1)$-connected and $C(n-1)$-connected, $m, n \geq 2$. If $X$ is a $C W$-complex of dimension $\leq m+n-2$, then the following sequence is exact

$$
[X, A] \stackrel{i_{*}}{\longrightarrow}[X, Y] \stackrel{p_{*}}{\longrightarrow}[X, C] .
$$

Now let $B$ be an $(r-1)$-connected space with $H_{r}(B)=G$, for $r \geq 2$. Then the homomorphism of the universal coefficient theorem for cohomology $\eta: H^{r}(B ; G) \rightarrow$ $\operatorname{Hom}\left(H_{r}(B), G\right)=\operatorname{Hom}(G, G)$ is an isomorphism.

Definition 2.2. The basic class $b^{r} \in H^{r}(B ; G)$ of $B$ is defined by $\eta\left(b^{r}\right)=i d$.

We can regard the basic class as a homotopy class $b^{r}: B \rightarrow K(G, r)$. Then $b^{r}$ is an $(r+1)$-equivalence, i.e., on homotopy groups it induces an isomorphism in dimensions $\leq r$ and an epimorphism in dimension $r+1$. Thus by [Sp, Cor. 23, p. 405], if $A$ is a $\mathrm{CW}$-complex of dimension $\leq r$, then $b_{*}^{r}:[A, B] \rightarrow[A, K(G, r)]=H^{r}(A ; G)$ is a bijection. Thus we have

Proposition 2.3. If $B$ is an $(r-1)$-connected space, $r \geq 2$ and $A$ is a $C W$-complex of dimension $\leq r$, then $g \in[A, B]$ is trivial if and only if $g^{*}\left(b^{r}\right)=0$.

We next consider homology decompositions. 
Definition 2.4. Given a 1-connected space $X$. A homology decomposition of $X$ consists of (i) a sequence of spaces $X_{n}, n \geq 2$ with $H_{i}\left(X_{n}\right)=0$ for $i>n$ and maps $j_{n}: X_{n} \rightarrow X$ such that $j_{n *}: H_{i}\left(X_{n}\right) \rightarrow H_{i}(X)$ is an isomorphism for $i \leq n$ and (ii) maps $k_{n}: M\left(H_{n+1}(X), n\right) \rightarrow X_{n}$ with $M\left(H_{n+1}(X), n\right) \stackrel{k_{n}}{\longrightarrow} X_{n} \stackrel{i_{n}}{\longrightarrow} X_{n+1}$ a mapping cone sequence (i.e., $X_{n+1}$ is the mapping cone of $k_{n}$ with inclusion $i_{n}$ ). We require that $j_{n+1} i_{n}=j_{n}: X_{n} \rightarrow X$. We refer to the collection of spaces and maps $\left\{X_{n} ; j_{n}, k_{n}, i_{n} ; n \geq 2\right\}$ as the homology decomposition of $X$. The space $X_{n}$ is called the nth homology section of $X$ and the maps $k_{n} \in \pi_{n}\left(H_{n+1}(X) ; X_{n}\right)$ the $n$th dual invariant.

We make several comments about this definition.

(1) For a 1-connected space of the homotopy type of a CW-complex, a homology decomposition always exists $\left[\mathrm{Hi}_{1}\right.$, Chaps. 8, 10].

(2) The dual invariant $k_{n}$ induces the trivial homomorphism on homology [ $\mathrm{Hi}_{1}$, p. 57].

(3) We can regard $X_{n}$ as a CW-complex of dimension $\leq n+1$ [ $\mathrm{Hi}_{1}$, p. 57].

(4) If $X$ is an $N$-dimensional CW-complex, then $j_{N}: X_{N} \rightarrow X$ is a homotopy equivalence and we can identify $X_{N}$ with $X$.

(5) If $H_{n+1}(X)=0$, then $M\left(H_{n+1}(X), n\right)=*$. Thus $k_{n}=0, X_{n+1}=X_{n}$ and $i_{n}=i d$. Note too that $X_{2}=M\left(H_{2}(X), 2\right)$.

We conclude this section by defining basic classes for a homology decomposition. Let $\left\{X_{n} ; j_{n}, k_{n}, i_{n} ; n \geq 2\right\}$ be a homology decomposition of $X$. Consider

$$
X_{r-1} \stackrel{j_{r-1}}{\longrightarrow} X \stackrel{q_{r}}{\longrightarrow} C_{r}
$$

where $C_{r}$ is the cofibre of $j_{r-1}$ and $q_{r}$ is the projection. Then $C_{r}$ is $(r-1)$-connected and $H_{r}\left(C_{r}\right) \approx H_{r}(X)$. Let $b^{r} \in H^{r}\left(C_{r} ; H_{r}(X)\right)$ be the basic class of $C_{r}$.

Definition 2.5. The element $h^{r}=q_{r}^{*}\left(b^{r}\right) \in H^{r}\left(X ; H_{r}(X)\right)$ is called the $r$ th basic class of the homology decomposition $\left\{X_{n} ; j_{n}, k_{n}, i_{n} ; n \geq 2\right\}$.

3. Induced maps. Given two spaces $X$ and $X^{\prime}$ with homology decompositions and a map $f: X \rightarrow X^{\prime}$. We consider when $f$ gives rise to induced maps, i.e., compatible maps of all the spaces of the homology decomposition of $X$ into the corresponding spaces of the homology decomposition of $X^{\prime}$.

Theorem 3.1. Let $X$ and $X^{\prime}$ have homology decompositions $\left\{X_{n} ; j_{n}, k_{n}, i_{n}\right\}$ and $\left\{X_{n}^{\prime} ; j_{n}^{\prime}, k_{n}^{\prime}, i_{n}^{\prime}\right\}$, respectively, and let $f: X \rightarrow X^{\prime}$ be a map.

(1) There is a map $f_{n}: X_{n} \rightarrow X_{n}^{\prime}$ such that $j_{n}^{\prime} f_{n}=f j_{n}$ if and only if $j_{n}^{*} f^{*}\left(h^{\prime n+1}\right)=0$ in $H^{n+1}\left(X_{n} ; H_{n+1}\left(X^{\prime}\right)\right)$, where $h^{\prime n+1}$ is the $(n+1)$ st basic class of the homology decomposition $\left\{X_{n}^{\prime} ; j_{n}^{\prime}, k_{n}^{\prime}, i_{n}^{\prime}\right\}$.

(2) Assume that $j_{r}^{*} f^{*}\left(h^{\prime} r+1\right)=0$ for $r=n, n+1$. Then there exists $f_{r}: X_{r} \rightarrow X_{r}^{\prime}$ such that $j_{r}^{\prime} f_{r}=f j_{r}$ for $r=n, n+1$ and $i_{n}^{\prime} f_{n}=f_{n+1} i_{n}$.

(3) Assume that there exists $f_{r}: X_{r} \rightarrow X_{r}^{\prime}$ for $r=n, n+1$ such that $i_{n}^{\prime} f_{n}=f_{n+1} i_{n}$. If $H_{n+1}(X)$ is free-abelian or if $X^{\prime}$ is 2-connected, then there exists $\hat{f}_{n}: M\left(H_{n+1}(X), n\right) \rightarrow$ $M\left(H_{n+1}\left(X^{\prime}\right), n\right)$ such that $k_{n}^{\prime} \hat{f}_{n}=f_{n} k_{n}$.

Proof. (1) If there is an $f_{n}: X_{n} \rightarrow X_{n}^{\prime}$ with $j_{n}^{\prime} f_{n}=f j_{n}$, then $j_{n}^{*} f^{*}\left(h^{\prime n+1}\right)=$ $f_{n}^{*} j_{n}^{*} q_{n+1}^{*}\left(b_{n+1}^{\prime}\right)=0$ since $q_{n+1}^{\prime} j_{n}^{\prime}=0$. Now suppose that $j_{n}^{*} f^{*}\left(h^{\prime} n+1\right)=0$. Then 
$\left(q_{n+1}^{\prime} f j_{n}\right)^{*}\left(b^{\prime n+1}\right)=0$. By Proposition $2.3, q_{n+1}^{\prime} f j_{n}=0$. Now apply Theorem 2.1 to the cofibration $X_{n}^{\prime} \stackrel{j_{n}^{\prime}}{\longrightarrow} X^{\prime} \stackrel{q_{n+1}^{\prime}}{\longrightarrow} C_{n+1}^{\prime}$ to conclude that

$$
\left[X_{n}, X_{n}^{\prime}\right] \stackrel{j_{n *}^{\prime}}{\longrightarrow}\left[X_{n}, X^{\prime}\right] \stackrel{q_{n+1 *}^{\prime}}{\longrightarrow}\left[X_{n}, C_{n+1}^{\prime}\right]
$$

is exact. Since $q_{n+1 *}^{\prime}\left(f j_{n}\right)=0$, there exists an $f_{n} \in\left[X_{n}, X_{n}^{\prime}\right]$ such that $f j_{n}=j_{n}^{\prime} f_{n}$.

(2) By (1), there exists $f_{n+1}: X_{n+1} \rightarrow X_{n+1}^{\prime}$ such that $f j_{n+1}=j_{n+1}^{\prime} f_{n+1}$. It suffices to prove that there is an $f_{n}: X_{n} \rightarrow X_{n}^{\prime}$ such that $i_{n}^{\prime} f_{n}=f_{n+1} i_{n}$. But this follows immediately from (1) by taking $\left\{X_{r} ; i_{n} \cdots i_{r}, k_{r}, i_{r} ; 2 \leq r \leq n\right\}$ and $\left\{X_{r}^{\prime} ; i_{n}^{\prime} \cdots i_{r}^{\prime}, k_{r}^{\prime}, i_{r}^{\prime} ; 2 \leq\right.$ $r \leq n\}$ as homology decompositions of $X_{n+1}$ and $X_{n+1}^{\prime}$, respectively.

(3) For notational convenience we write $H_{i}=H_{i}(X)$ and $H_{i}^{\prime}=H_{i}\left(X^{\prime}\right)$. Here we consider the cofibre sequence

$$
M\left(H_{n+1}^{\prime}, n\right) \stackrel{k_{n}^{\prime}}{\longrightarrow} X_{n}^{\prime} \stackrel{i_{n}^{\prime}}{\longrightarrow} X_{n+1}^{\prime} .
$$

If $H_{n+1}$ is free-abelian so that $\operatorname{dim} M\left(H_{n+1}, n\right) \leq n$ or if $X^{\prime}$ is 2-connected so that $X_{n+1}^{\prime}$ is 2-connected, then by Theorem 2.1 the following sequence is exact

$$
\left[M\left(H_{n+1}, n\right), M\left(H_{n+1}^{\prime}, n\right)\right] \stackrel{k_{n *}^{\prime}}{\longrightarrow}\left[M\left(H_{n+1}, n\right), X_{n}^{\prime}\right] \stackrel{i_{n *}^{\prime}}{\longrightarrow}\left[M\left(H_{n+1}, n\right), X_{n+1}^{\prime}\right] .
$$

But $i_{n *}^{\prime}\left(f_{n} k_{n}\right)=f_{n+1} i_{n} k_{n}=0$. Thus there is an $\hat{f}_{n} \in\left[M\left(H_{n+1}, n\right), M\left(H_{n+1}^{\prime}, n\right)\right]$ such that $k_{n}^{\prime} \hat{f}_{n}=f_{n} k_{n}$.

Remarks 3.2. (1) Part (1) of Theorem 3.1 was proved in $\left[\mathrm{Cu}_{1}\right]$, though we have given a different proof based on Theorem 2.1. It would be interesting to know if (3) holds under weaker hypotheses. We note that there is considerable freedom in the choice of $\hat{f}_{n}$ in (3), e.g., if $k_{n}^{\prime}=0$, then any map $M\left(H_{n+1}, n\right) \rightarrow M\left(H_{n+1}^{\prime}, n\right)$ can be taken for $\hat{f}_{n}$.

(2) There are a few cases in which induced maps always exist, i.e., when (1) of Theorem 3.1 holds. We mention two of these: (i) If $X^{\prime}$ is a rational space, then $H_{r}\left(X^{\prime}\right)$ is a rational vector space for all $r$. Thus $\operatorname{Ext}\left(H_{r}\left(X_{r}\right), H_{r+1}\left(X^{\prime}\right)\right)=0$ for all $r$. But $j_{r}^{*} f^{*}\left(h^{\prime r+1}\right) \in \operatorname{Ext}\left(H_{r}\left(X_{r}\right), H_{r+1}\left(X^{\prime}\right)\right)$. Hence in this case there are maps $f_{n}: X_{n} \rightarrow X_{n}^{\prime}$ which satisfy (1) and (2) of Theorem 3.1 for all $n$. (ii) If $f: X \rightarrow X$ is such that $f^{*}=$ id $: H^{n+1}\left(X ; H_{n+1}(X)\right) \rightarrow H^{n+1}\left(X ; H_{n+1}(X)\right)$, then $j_{n}^{*} f^{*}\left(h^{n+1}\right)=j_{n}^{*} q_{n+1}^{*}\left(b^{n+1}\right)=0$. Thus there is an $f_{n}: X_{n} \rightarrow X_{n}$ such that $j_{n} f_{n}=f j_{n}$.

Next we give a concrete example to show that induced maps do not always exist.

ExAmple 3.3. Let $T$ be a non-trivial finite abelian group and $F$ a non-trivial freeabelian group of finite rank. Let $n \geq 3, M_{1}=M(T, n-1), M_{2}=M(F, n)$ and $X=$ $X^{\prime}=M_{1} \vee M_{2}$. Let $\lambda_{s}: M_{s} \rightarrow X$ be the inclusions and $\pi_{r}: X \rightarrow M_{r}$ the projections, $r, s=1,2$. A map $f: X \rightarrow X$ is completely determined by the 4-tuple $\left(f_{11}, f_{12}, f_{21}, f_{22}\right)$, where $f_{r s}: M_{s} \rightarrow M_{r}$ is defined by $f_{r s}=\pi_{r} f \lambda_{s}$ (see for example [A-M, §4]). A homology decomposition for $X$ is obtained by setting $X_{2}=\cdots=X_{n-2}=*, X_{n-1}=M_{1}$ and $X_{n}=X=M_{1} \vee M_{2}$. Then $i_{n-1}: X_{n-1} \rightarrow X_{n}$ is the inclusion $\lambda_{1}: M_{1} \rightarrow M_{1} \vee M_{2}$. Suppose that $f$ induces $f^{\prime}: M_{1} \rightarrow M_{1}$ such that $\lambda_{1} f^{\prime}=f \lambda_{1}$. If $x \in M_{1}$,

$$
\left(f^{\prime}(x), *\right)=\lambda_{1} f^{\prime}(x)=f \lambda_{1}(x)=\left(f_{11}(x), f_{21}(x)\right) .
$$


Hence $f_{11}=f^{\prime}$ and $f_{21}=0$. Thus if $f: X \rightarrow X$ is a map such that $f_{21} \neq 0$, then there can be no map $f^{\prime}=f_{n-1}: X_{n-1} \rightarrow X_{n-1}$ such that $i_{n-1} f_{n-1}=f i_{n-1}$. But $f_{21} \in\left[M_{1}, M_{2}\right]=\pi_{n-1}(T ; M(F, n)) \approx \operatorname{Ext}(T, F) \neq 0$ since $T$ and $F$ are non-trivial. Therefore we can choose $f_{21} \neq 0$. For example, if $T=\mathbb{Z}_{m}$ and $F=\mathbb{Z}$, then $f_{21}$ can be taken to be the projection $M\left(\mathbb{Z}_{m}, n-1\right) \rightarrow S^{n}$. We then form $f=\left(f_{11}, f_{12}, f_{21}, f_{22}\right)$ which admits no induced map of homology decompositions.

We conclude this section with a number of simple results which are a direct consequence of the existence of induced maps.

It is known that for a fixed $n$, the homotopy type of the $n$th homology section of a homology decomposition of $X$ is not determined by $X$. More precisely, an example is given in [B-C, §3] of two spaces $X$ and $X^{\prime}$ with $n$th homology sections $X_{n}$ and $X_{n}^{\prime}$ such that $X$ and $X^{\prime}$ have the same homotopy type but $X_{n}$ and $X_{n}^{\prime}$ do not. We next give a condition which ensures that this does not happen. This generalizes Theorems 3.3 and 3.4 of $[\mathrm{B}-\mathrm{C}]$.

Proposition 3.4. Let $\left\{X_{n} ; j_{n}, k_{n}, i_{n}\right\}$ and $\left\{X_{n}^{\prime} ; j_{n}^{\prime}, k_{n}^{\prime}, i_{n}^{\prime}\right\}$ be homology decompositions of $X$ and $X^{\prime}$ respectively. If $f: X \rightarrow X^{\prime}$ is a homotopy equivalence and $\operatorname{Ext}\left(H_{n}(X), H_{n+1}\left(X^{\prime}\right)\right)=0$, then there exists a homotopy equivalence $f_{n}: X_{n} \rightarrow X_{n}^{\prime}$ such that $f j_{n}=j_{n}^{\prime} f_{n}$. If in addition, $\operatorname{Ext}\left(H_{n+1}(X), H_{n+2}\left(X^{\prime}\right)\right)=0$ and either $X^{\prime}$ is 2-connected or $H_{n+1}(X)$ is free-abelian, then there exists a homotopy equivalence $\hat{f}_{n}: M\left(H_{n+1}(X), n\right) \rightarrow M\left(H_{n+1}\left(X^{\prime}\right), n\right)$ such that $k_{n}^{\prime} \hat{f}_{n}=f_{n} k_{n}$.

Proof. Since $H^{n+1}\left(X_{n} ; H_{n+1}\left(X^{\prime}\right)\right) \approx \operatorname{Ext}\left(H_{n}(X), H_{n+1}\left(X^{\prime}\right)\right)=0, j_{n}^{*} f^{*}\left(h_{n+1}^{\prime}\right)=$ 0 . Thus there exists $f_{n}: X_{n} \rightarrow X_{n}^{\prime}$ with $f j_{n}=j_{n}^{\prime} f_{n}$. It follows that $f_{n}$ induces an isomorphism of homology, and so is a homotopy equivalence.

Similarly the condition $\operatorname{Ext}\left(H_{n+1}(X), H_{n+2}\left(X^{\prime}\right)\right)=0$ implies the existence of a homotopy equivalence $f_{n+1}: X_{n+1} \rightarrow X_{n+1}^{\prime}$ such that $f_{n+1} i_{n}=i_{n}^{\prime} f_{n}$. Also the condition $X^{\prime}$ is 2-connected or $H_{n+1}(X)$ is free-abelian implies there exists $\hat{f}_{n}: M\left(H_{n+1}(X), n\right) \rightarrow$ $M\left(H_{n+1}\left(X^{\prime}\right), n\right)$ with $k_{n}^{\prime} \hat{f}_{n}=f_{n} k_{n}$. It follows that the diagram

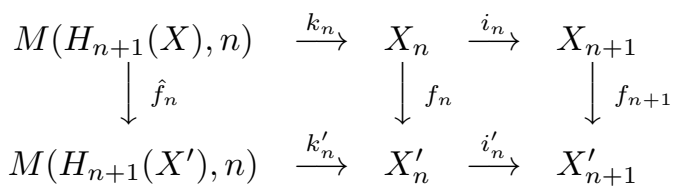

commutes. Thus $\hat{f}_{n}$ is a homotopy equivalence.

We next determine, under suitable restrictions, the homotopy types of all $(n+1)$ st homology sections with fixed $n$th homology section. We first introduce some notation. If $A$ and $B$ are spaces, then define an equivalence relation on the set $[A, B]$ as follows: if $f, g \in[A, B]$, then $f$ is equivalent to $g$ means that there exist homotopy equivalences $a: A \rightarrow A$ and $b: B \rightarrow B$ such that $g=b f a$. We let $[[A, B]]$ denote the set of equivalence classes. We consider the set of homotopy types of mapping cones of maps from a Moore space to a homology section.

Proposition 3.5. Let $B$ be a space such that $H_{i}(B)=0$ for $i>n, n \geq 2$, and consider the collection of maps $f: M(G, m) \rightarrow B$ for a fixed abelian group $G$ and integer 
$m \geq n$ (if $m=n$, we require that $f_{*}=0$ in homology). Then the set of homotopy types of mapping cones $C_{f}$ for all such $f$ is in one-one correspondence with the set $[[M(G, m), B]]$ provided $\operatorname{Ext}\left(H_{m}(B), G\right)=0$ and either $B$ is 2-connected or $G$ is free-abelian.

Proof. We have a homology decomposition of $X=C_{f}$ with $X_{m}=B, k_{m}=f$ : $M(G, m) \rightarrow B$ and $X_{m+1}=X$. Let $X^{\prime}=C_{g}$ be another such mapping cone with analogous homology decomposition and assume that $C_{f}$ and $C_{g}$ have the same homotopy type. By Proposition 3.4, there exist homotopy equivalences $a: M(G, m) \rightarrow M(G, m)$ and $b: B \rightarrow B$ such that $b f=g a$. Thus $f$ is equivalent to $g$.

Conversely, if $f, g: M(G, m) \rightarrow B$ are equivalent, then it is easily seen that $C_{f}$ and $C_{g}$ have the same homotopy type.

Corollary 3.6. Let $B$ be a space such that $H_{i}(B)=0$ for $i>n$ and $G$ an abelian group. Suppose $\operatorname{Ext}\left(H_{n}(B), G\right)=0$ and either $B$ is 2-connected or $G$ is free-abelian. Then the set of homotopy types of $(n+1)$ st homology sections with nth homology section $B$ and $(n+1)$ st homology group $G$ is in one-one correspondence with the equivalence classes of homologically trivial maps in $[[M(G, n), B]]$.

REMARK 3.7. Corollary 3.6 generalizes Theorem 4.2 of [B-C]. The dual result for Postnikov sections is true without any restrictions $\left[\mathrm{Ar}_{1}, 5.2\right.$, p. 197].

4. Co-H-spaces. We first recall the definitions of co-H-space and co-H-map (for more details, see $\left.\left[\mathrm{Ar}_{2}\right]\right)$. If $X$ is a space, then $\phi: X \rightarrow X \vee X$ is called a comultiplication if $q_{i} \phi=i d: X \rightarrow X$, where $q_{i}: X \vee X \rightarrow X$ are the projections, $i=1,2$. The pair $(X, \phi)$ is then called a co-H-space. If $(A, \psi)$ and $(X, \phi)$ are co-H-spaces, and $f: A \rightarrow X$ is a map, then $f$ is called a co-H-map if $\phi f=(f \vee f) \psi$. We then write $f:(A, \psi) \rightarrow(X, \phi)$. If $f$ is a co-H-map and a homotopy equivalence, we say that $f$ is a co-H-equivalence and that the co-H-spaces $A$ and $X$ are co-H-equivalent.

The following lemma will be useful.

Lemma 4.1. Given spaces $A$ and $X$, maps $f: A \rightarrow X, \phi^{\prime}: A \rightarrow A \vee A$ and $\phi:$ $X \rightarrow X \vee X$ and projections $p_{i}: A \vee A \rightarrow A, i=1,2$. Suppose $(X, \phi)$ is a co-H-space, $(f \vee f) \phi^{\prime}=\phi f: A \rightarrow X \vee X$ and $p_{i} \phi^{\prime}: A \rightarrow A$ are homotopy equivalences. Then there exists a comultiplication $\psi: A \rightarrow A \vee A$ such that $f:(A, \psi) \rightarrow(X, \phi)$ is a co-H-map.

Proof. Consider the commutative diagram



Let $a_{i}=p_{i} \phi^{\prime}: A \rightarrow A$ and let $\bar{a}_{i}: A \rightarrow A$ be the homotopy inverse of $a_{i}$. Then $f a_{i}=q_{i} \phi f=f$ and so $f=f \bar{a}_{i}$. Now define $\psi=\left(\bar{a}_{1} \vee \bar{a}_{2}\right) \phi^{\prime}: A \rightarrow A \vee A$. Then

$$
p_{i} \psi=\bar{a}_{i} p_{i} \phi^{\prime}=\bar{a}_{i} a_{i}=i d
$$

for $i=1,2$. Thus $\psi$ is a comultiplication of $A$. Finally,

$$
(f \vee f) \psi=\left(f \bar{a}_{1} \vee f \bar{a}_{2}\right) \phi^{\prime}=(f \vee f) \phi^{\prime}=\phi f
$$

and so $f:(A, \psi) \rightarrow(X, \phi)$ is a co-H-map. 
Next we consider a space $X$ with homology decomposition $\left\{X_{n} ; j_{n}, k_{n}, i_{n}\right\}$. It is clear that $\left\{X_{n} \vee X_{n} ; j_{n} \vee j_{n}, k_{n} \vee k_{n}, i_{n} \vee i_{n}\right\}$ is a homology decomposition of $X \vee X$. We express the basic classes of this homology decomposition of $X \vee X$ in terms of the basic classes of the homology decomposition of $X$. Let $q_{1}, q_{2}: X \vee X \rightarrow X$ be projections and let $i_{1}, i_{2}: X \rightarrow X \vee X$ be inclusions. For any space $A$, denote by $i_{r * *}: H^{k}\left(A ; H_{l}(X)\right) \rightarrow$ $H^{k}\left(A ; H_{l}(X \vee X)\right), r=1,2$, the coefficient homomorphism induced by $i_{r *}: H_{l}(X) \rightarrow$ $H_{l}(X \vee X)$. Let $h^{n} \in H^{n}\left(X ; H_{n}(X)\right)$ be the $n$th basic class of the homology decomposition of $X$. Then it is straightforward to show that

$$
\chi^{n}=i_{1 * *} q_{1}^{*}\left(h^{n}\right)+i_{2 * *} q_{2}^{*}\left(h^{n}\right) \in H^{n}\left(X \vee X ; H_{n}(X \vee X)\right)
$$

is the $n$th basic class of the homology decomposition of $X \vee X$.

Now we consider the homology decomposition of a co-H-space.

THEOREM 4.2. If $(X, \phi)$ is a co-H-space and $\left\{X_{n} ; j_{n}, k_{n}, i_{n}\right\}$ a homology decomposition of $X$, then there are comultiplications $\phi_{n}: X_{n} \rightarrow X_{n} \vee X_{n}$ such that $j_{n}:\left(X_{n}, \phi_{n}\right) \rightarrow$ $(X, \phi)$ and $i_{n}:\left(X_{n}, \phi_{n}\right) \rightarrow\left(X_{n+1}, \phi_{n+1}\right)$ are co-H-maps. If in addition $X$ is 2-connected or $H_{n+1}(X)$ is free-abelian, then $k_{n}:\left(M\left(H_{n+1}(X), n\right), \mu_{n}\right) \rightarrow\left(X_{n}, \phi_{n}\right)$ is a co-H-map, where $\mu_{n}$ is the canonical comultiplication of the Moore space $M\left(H_{n+1}(X), n\right)$.

Proof. We verify (1) of Theorem 3.1 for the map $\phi$ and basic class $\chi^{n+1}$. We have

$$
\begin{aligned}
j_{n}^{*} \phi^{*}\left(\chi^{n+1}\right) & =j_{n}^{*} \phi^{*}\left(i_{1 * *} q_{1}^{*}\left(h^{n+1}\right)+i_{2 * *} q_{2}^{*}\left(h^{n+1}\right)\right) \\
& =i_{1 * *} j_{n}^{*}\left(q_{1} \phi\right)^{*} q_{n+1}^{*}\left(b^{n+1}\right)+i_{2 * *} j_{n}^{*}\left(q_{2} \phi\right)^{*} q_{n+1}^{*}\left(b^{n+1}\right) \\
& =i_{1 * *} j_{n}^{*} q_{n+1}^{*}\left(b^{n+1}\right)+i_{2 * *} j_{n}^{*} q_{n+1}^{*}\left(b^{n+1}\right)=0
\end{aligned}
$$

since $q_{n+1} j_{n}=0$. Thus there exists $\phi_{n}^{\prime}: X_{n} \rightarrow X_{n} \vee X_{n}$ such that $\left(j_{n} \vee j_{n}\right) \phi_{n}^{\prime}=\phi j_{n}$ and $\left(i_{n} \vee i_{n}\right) \phi_{n}^{\prime}=\phi_{n+1} i_{n}$. Then with $p_{r}: X_{n} \vee X_{n} \rightarrow X_{n}$ the projections,

$$
j_{n} p_{r} \phi_{n}^{\prime}=q_{r}\left(j_{n} \vee j_{n}\right) \phi_{n}^{\prime}=q_{r} \phi j_{n}=j_{n} .
$$

But $j_{n *}: H_{i}\left(X_{n}\right) \rightarrow H_{i}(X)$ is a monomorphism for all $i$. Therefore $p_{r} \phi_{n}^{\prime}$ is a homotopy equivalence. By Lemma 4.1, there exists a comultiplication $\phi_{n}: X_{n} \rightarrow X_{n} \vee X_{n}$ such that $\left(j_{n} \vee j_{n}\right) \phi_{n}=\phi j_{n}$. From the construction of $\phi_{n}$ in Lemma 4.1, it immediately follows that $\left(i_{n} \vee i_{n}\right) \phi_{n}=\phi_{n+1} i_{n}$.

Now assume that $X$ is 2-connected or $H_{n+1}(X)$ is free-abelian and write $M_{n}$ for $M\left(H_{n+1}(X), n\right)$. Then there exists $\hat{\phi}_{n}: M_{n} \rightarrow M_{n} \vee M_{n}$ such that $\left(k_{n} \vee k_{n}\right) \hat{\phi}_{n}=\phi_{n} k_{n}$. Let $r_{i}: M_{n} \vee M_{n} \rightarrow M_{n}, p_{i}: X_{n} \vee X_{n} \rightarrow X_{n}$ and $q_{i}: X_{n+1} \vee X_{n+1} \rightarrow X_{n+1}$ be projections and consider the commutative diagram

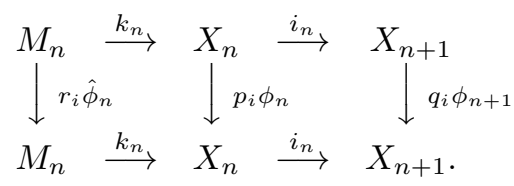

Since $p_{i} \phi_{n}=i d$ and $q_{i} \phi_{n+1}=i d$, it follows that $r_{i} \hat{\phi}_{n}$ is a homotopy equivalence. By Lemma 4.1 , there exists a comultiplication $\mu_{n}$ of $M_{n}$ such that $k_{n}:\left(M_{n}, \mu_{n}\right) \rightarrow\left(X_{n}, \phi_{n}\right)$ is a co-H-map. Then $\mu_{n}$ is the canonical comultiplication of $M_{n}$ (see Remark $4.4(2)$ ).

Corollary 4.3. If $X$ is a co-H-space and $\left\{X_{n} ; j_{n}, k_{n}, i_{n}\right\}$ is a homology decomposition of $X$, then there is a comultiplication on each nth homology section $X_{n}$ such that 
$j_{n}: X_{n} \rightarrow X$ and $i_{n}: X_{n} \rightarrow X_{n+1}$ are co-H-maps. If either $X$ is 2-connected or $X$ has no torsion in its homology, then all dual invariants $k_{n}: M\left(H_{n+1}(X), n\right) \rightarrow X_{n}$ are co-H-maps.

REMARKS 4.4. (1) The first assertion of Corollary 4.3 was originally proved in $\left[\mathrm{Cu}_{2}\right.$, Lem. 2.3] (see also $\left[\mathrm{B}-\mathrm{H}_{1}\right]$ and $[\mathrm{G}-\mathrm{K}]$ ). Moreover, Berstein and Hilton proved in $\left[\mathrm{B}-\mathrm{H}_{1}\right.$, $\S 3]$ the analogous result for spaces of cat $\leq n$. The second assertion of Corollary 4.3 for 2-connected spaces was proved by Golasiński and Klein in [G-K, Cor. 3] by different methods.

(2) A Moore space $M(G, n)$ has a unique comultiplication for $n \geq 3$. However, the comultiplications on $M(G, 2)$ are in one-one correspondence with $\operatorname{Ext}(G, G \otimes G)$. Thus if $G$ is free-abelian, $M(G, 2)$ has a unique comultiplication. For more details on comultiplications on Moore spaces, see $[\mathrm{A}-\mathrm{G}]$.

(3) It would be interesting to know if the second assertion of Corollary 4.3 is true with a weaker hypothesis or even without any restrictions. In this connection we note that the first dual invariant of a co-H-space is always a co-H-map. Because this result is limited and the proof is long, we just state it: Let $(X, \phi)$ be a co-H-space, $\left\{X_{n} ; j_{n}, k_{n}, i_{n}\right\}$ a homology decomposition of $X$ and $\phi_{n}: X_{n} \rightarrow X_{n} \vee X_{n}$ the induced comultiplication. Then there exists a comultiplication $\psi_{2}$ on $M\left(H_{3}(X), 2\right)$ such that $k_{2}:\left(M\left(H_{3}(X), 2\right), \psi_{2}\right) \rightarrow\left(X_{2}, \phi_{2}\right)$ is a co-H-map.

We conclude the paper by giving a new proof of a basic result on co-H-spaces which is due to Berstein and Hilton. We base our proof on Theorem 4.2 and another result of Berstein and Hilton which we now state.

Theorem $\mathrm{B}\left[\mathrm{B}-\mathrm{H}_{2}\right.$, Thm. B]. If $A$ and $B$ are spaces such that dimension $A \leq 3 q-2$ and $B$ is $(q-1)$-connected, $q \geq 1$, then every co-H-map $\Sigma A \rightarrow \Sigma B$ is a suspension.

The following theorem appears in $\left[\mathrm{B}-\mathrm{H}_{2}\right.$, Thm. A].

TheOREM 4.5. If $X$ is a $(q-1)$-connected $C W$-complex of dim $\leq 3 q-3, q \geq 1$, and $\phi$ is a comultiplication of $X$, then $(X, \phi)$ is co-H-equivalent to a suspension.

Proof. For notational convenience we write $H_{i}$ for $H_{i}(X)$. The case $q=1$ is trivial and so we first consider the case $q=2$. Then $X$ is a 1-connected complex of dimension $\leq 3$. Thus $H_{3}$ is free-abelian and so by Theorem 4.2, $k_{2}: M\left(H_{3}, 2\right) \rightarrow X_{2}=M\left(H_{2}, 2\right)$ is a co-H-map (this also follows from the result stated in Remark 4.4 (3)). By Theorem B above, $k_{2}$ is a suspension, and so $X=X_{3}$ is co-H-equivalent to a suspension. Now assume $q \geq 3$ so that $X$ is 2 -connected. We let $\left\{X_{n} ; j_{n}, k_{n}, i_{n}\right\}$ be a homology decomposition for $X$ with $X_{q-1}=*, X_{q}=M\left(H_{q}, q\right)$ and $X_{3 q-3}=X$. Then by Corollary 4.3, all $X_{n}$ are co-H-spaces and all $j_{n}, i_{n}$ and $k_{n}$ are co-H-maps. We prove by induction on $i$ that $X_{i}$ is a suspension, $i=q, \ldots, 3 q-3$. Clearly this is true for $i=q$. Now suppose that $X_{i}=\Sigma X_{i}^{\prime}$ for some space $X_{i}^{\prime}$ and consider $k_{i}: \Sigma M\left(H_{i+1}, i-1\right) \rightarrow \Sigma X_{i}^{\prime}$. If $i<3 q-4$, then $\operatorname{dim} M\left(H_{i+1}, i-1\right) \leq i \leq 3 q-5$. Now let $i=3 q-4$. Then $H_{i+1}=H_{3 q-3}$ is free-abelian, and so $\operatorname{dim} M\left(H_{3 q-3}, 3 q-5\right) \leq 3 q-5$. Thus $\operatorname{dim} M\left(H_{i+1}, i-1\right) \leq 3 q-5$ for all $i \leq 3 q-4$. Therefore we apply Theorem B to conclude that $k_{i}$ is a suspension and so $X_{i+1}$ is co-H-equivalent to a suspension. This completes the induction. 


\section{References}

[Ar 1 M. Arkowitz, The group of self-homotopy equivalences - A survey, Groups of SelfHomotopy Equivalences and Related Topics, Lecture Notes in Math. 1425, SpringerVerlag 1990, 170-203.

[Ar 2 M. Arkowitz, Co-H-spaces, Handbook of Algebraic Topology, Elsevier Science, North Holland, 1995, 1143-1173.

[A-G] M. Arkowitz and M. Golasiński, Co-H-structures on Moore spaces of type $(G, 2)$, Can. J. of Math. 46 (1994), 673-686.

[A-M] M. Arkowitz and K. Maruyama, Self homotopy equivalences which induce the identity on homology, cohomology or homotopy groups, Topology Appl. (to appear).

[B- $\left.\mathrm{H}_{1}\right] \quad$ I. Berstein and P. Hilton, Category and generalized Hopf invariants, Ill. J. of Math. 4 (1960), 437-451.

[B-H $\left.{ }_{2}\right]$ I. Berstein and P. Hilton, On suspensions and comultiplications, Topology 2 (1963), 73-82.

[B-C] E. Brown and A. Copeland, An homology analogue of Postnikov systems, Mich. Math. J. 6 (1959), 313-330.

$\left[\mathrm{Cu}_{1}\right]$ C. Curjel, On the homology decomposition of polyhedra, Ill. J. of Math. 7 (1963), 121-136.

$\left[\mathrm{Cu}_{2}\right] \quad$ C. Curjel, A note on spaces of category $\leq 2$, Math. Zeit. 80 (1963), 293-299.

[G-K] M. Golasiński and J. Klein, On maps into a co-H-space, (preprint).

$\left[\mathrm{Hi}_{1}\right] \quad$ P. Hilton, Homotopy and Duality, Gordon and Breach, 1965.

$\left[\mathrm{Hi}_{2}\right]$ P. Hilton, On excision and principal fibrations, Comm. Math. Helv. 35 (1961), 77-84.

[Sp] E. Spanier, Algebraic Topology, McGraw-Hill, 1966.

[Wh] G. Whitehead, Elements of Homotopy Theory, Graduate Texts in Math. 61, SpringerVerlag (1978). 